\title{
Formulation and clinical evaluation of silymarin pluronic-lecithin organogels for treatment of atopic dermatitis
}

This article was published in the following Dove Press journal:

Drug Design, Development and Therapy

10 March 2016

Number of times this article has been viewed

\author{
Fatma M Mady ${ }^{1,2}$ \\ Hanaa Essa ${ }^{2}$ \\ Tarek El-Ammawi ${ }^{3}$ \\ Hamdy Abdelkader ${ }^{2}$ \\ Amal K Hussein ${ }^{2}$ \\ 'Department of Pharmaceutics and \\ Pharmaceutical Technology, Faculty \\ of Pharmacy, Taibah University, \\ Medina, Saudi Arabia; ${ }^{2}$ Department of \\ Pharmaceutics, Faculty of Pharmacy, \\ Minia University, Minia, Egypt; \\ ${ }^{3}$ Department of Dermatology, STDs, \\ and Andrology, Minia University \\ Hospital, Minia, Egypt
}

\begin{abstract}
Silymarin is a naturally occurring flavonoid drug; evidence from recent research has highlighted its use as a potential treatment for atopic dermatitis (AD). Both poor water solubility and drug permeability have hindered the percutaneous absorption of silymarin. Formulation of silymarin into pluronic-lecithin organogel (PLO) basis for topical skin delivery is the main aim of this work. Six different PLO formulations were prepared containing various pluronic to lecithin ratios using two cosolvent systems of ethyl alcohol and dimethyl sulfoxide. Formulation 2 (20\% pluronic and 3\% lecithin) was found to be the optimal base for topical delivery of silymarin as it showed optimum $\mathrm{pH}$, viscosity, drug content, and satisfactory in vitro silymarin permeation. The silymarin PLO formulation significantly relieved inflammatory symptoms of AD such as redness, swelling, and inflammation. These findings warrant the ability for application of these novel silymarin PLO formulations as a novel treatment for AD.
\end{abstract}

Keywords: silymarin, pluronic lecithin organogel, atopic dermatitis, skin penetration

\section{Introduction}

Atopic dermatitis (AD) is a chronically relapsing inflammatory disease; AD is characterized by intensely pruritic skin and occurs quite often in children. ${ }^{1}$ This is associated with increased production of $\mathrm{IgE}$ and/or altered pharmacological reactivity. ${ }^{2}$ The characteristic symptoms of AD include skin dryness, erythema, oozing, and it is occasionally followed by crusting and lichenification of the skin in untreated patients. Pruritus and skin itching are the most annoying symptoms and are responsible for much of the disease burden for patients and their families. ${ }^{3}$ Therefore, the main goal of the management of AD patients is based on the use of topical anti-inflammatory preparations along with optimum hydration of the skin. However, some patients may require phototherapy or systemic treatment in severe cases. ${ }^{4,5}$

Treatment of acute flare-ups is still based on topical glucocorticosteroids. ${ }^{6}$ Relapse after the discontinuation of oral steroids is well known. Long-term therapy of corticosteroids is associated with a series of well-documented side effects such as: increased risk of glaucoma and cataracts, ${ }^{7}$ in addition to the high incidence of steroid addiction when they are withdrawn. ${ }^{8}$

Recently, there has been a growing interest in using herbal medicines for the treatment of skin disease..$^{9-12}$

Silymarin is a natural polyphenolic flavonoid derived from milk thistle seeds. It has long been used as a traditional herbal hepatoprotective agent. ${ }^{13}$ It is one of the most widely used compounds of flavonoids because of its extensive therapeutic properties.
Correspondence: Amal K Hussein

Department of Pharmaceutics,

Faculty of Pharmacy, Minia University,

Minia 61519, Egypt

Tel $+2010205|05|$

Fax +20862369075

Email amal.hussein@mu.edu.eg
Drug Design, Development and Therapy 2016:10 II01-1110

(c) (1) (8) ๑ 2016 Mady et al. This work is published and licensed by Dove Medical Press Limited. The full terms of this license are available at https:/www.dovepress.com/terms.php cc) hereby accept the Terms. Non-commercial uses of the work are permitted without any further permission from Dove Medical Press Limited, provided the work is properly attributed. For permision for commercial use of this work, please see paragraphs 4.2 and 5 of our Terms (https://www.doveppess. com/terms.php).
Dovepress

http://dx.doi.org/1 0.2147/DDDT.S103423 
Zhao et al reported that silymarin has a diverse effect on the skin with chemoprotective and anticancer properties. ${ }^{10}$ Also, it was found that silymarin plays a role in the prevention of ultraviolet-B-induced skin damage, with a skin whitening effect. ${ }^{11,14}$ Moreover, silymarin has a positive effect on wound healing and skin burns owing to its anti-inflammatory and antioxidant effects. ${ }^{15-17}$ In addition, Han et al demonstrated that silymarin can exert a suppressive effect on chemically induced irritant contact dermatitis in an animal model. Furthermore, it has been reported that silymarin might inhibit the expression of cytokines and infiltration of neutrophils, therefore, it has a potential role for prevention and treatment of cutaneous inflammatory diseases. ${ }^{18}$ It was also found that prophylactic use of silymarin-based cream was associated with both significant decrease in the duration, and maximum severity of dermatitis compared with the standard of care treatment. Therefore, silymarin-based cream is a promising candidate for a safe prophylactic treatment option of radiodermatitis. ${ }^{19}$ It is well known that the stratum corneum is considered the major obstacle for topical delivery, which controls the drug transport even through the most superficial layers of the epidermis. ${ }^{20}$ Limited skin permeability of silymarin results from its lipophilicity and limited solubility. Hence, ideal topical drugs must possess both lipoidal and aqueous solubility. ${ }^{21}$ Spada et al studied the influence of silymarin complexation with cyclodextrin and formulation into oil-in-water emulsion on permeation in porcine skin. The penetration of the silymarin into the stratum corneum was successfully achieved using the phytocomplex; approximately $80 \%$ of silymarin was absorbed by the skin within 1 hour, compared with almost $30 \%$ permeability reported with uncomplexed silymarin. ${ }^{22}$

Recently, pluronic-lecithin organogels (PLOs) have gained popularity as transdermal and topical drug delivery systems owing to their biphasic composition and versatility; PLO can enhance solubility of poorly soluble drugs and enhance penetrability of hydrophilic drugs. ${ }^{23}$

Therefore, this work aimed to formulate and evaluate silymarin PLO as a novel treatment for AD. Specific objectives will look at: studying the effect of different parameters (polymer type and oily phase) on both the drug release and skin permeability. The efficacy of the formulated PLO loaded silymarin for treatment of AD patients was also studied.

\section{Materials and methods Materials}

Silymarin was a generous gift from SEDICO Pharmaceutical Company (Cairo, Egypt). Lecithin was purchased from
MP Biomedicals (LCC, Santa Ana, CA, USA). Isopropyl myristate (IPM), pluronic F-127, and dimethyl sulfoxide (DMSO) were purchased from Sigma-Aldrich Co. (St Louis, MO, USA). Potassium dihydrogen orthophosphate, disodium hydrogen orthophosphate, and sodium chloride were purchased from ADWIC (El-Nasr Pharmaceutical Co., Cairo, Egypt).

\section{Methods}

\section{Analytical method validation of silymarin}

A spectrophotometric method was developed and validated for determination of silymarin. Validation parameters included linearity, accuracy, and precision. The spectrophotometric method was validated by measuring absorbance at different concentration ranges and plotting calibration curves. The average accuracy and standard deviation (SD) were calculated. Precision was validated by measuring absorbance at six concentrations. Precision was expressed by the SD and relative $\mathrm{SD}$ of measured response.

\section{Preparation of silymarin PLO gels}

Different PLO formulations were prepared and Table 1 shows the composition of the different PLO formulations. The oil phase, containing soya lecithin was prepared by dissolving its components in an appropriate quantity of IPM overnight at room temperature. For preparing the aqueous phase, the calculated amounts of pluronic F-127 and ascorbic acid were dissolved in cold water and stored in a refrigerator (at $4^{\circ} \mathrm{C}$ ). Silymarin was dissolved in either ethyl alcohol or DMSO, and mixed with the prepared aqueous phase. The aqueous phase $(80 \%)$ was slowly added drop-wise to the oil phase $(20 \%)$ with continuous stirring $(1,000 \mathrm{rpm})$ for 1 minute at room temperature. The $\mathrm{pH}$ of the final formulated organogels was recorded using a digital $\mathrm{pH}$ meter (HANNA Instruments, Woonsocket, RI, USA).

Table I Composition of different PLO gels loaded with silymarin

\begin{tabular}{llllllll}
\hline Ingredients & \multicolumn{1}{l}{ Formulation code } \\
\cline { 2 - 9 } & FI & F2 & F3 & F4 & F5 & F6 & F2* \\
\hline Silymarin (gm) & 0.2 & 0.2 & 0.2 & 0.2 & 0.2 & 0.2 & 0.2 \\
Lecithin (gm) & 3 & 3 & 3 & 5 & 7 & 9 & 3 \\
Isopropyl myristate $(\mathrm{mL})$ & 100 & 100 & 100 & 100 & 100 & 100 & 100 \\
Pluronic I27 (gm) & 10 & 20 & 30 & 20 & 20 & 30 & 20 \\
Water (mL) & 100 & 100 & 100 & 100 & 100 & 100 & 100 \\
Ascorbic acid (gm) & 0.2 & 0.2 & 0.2 & 0.2 & 0.2 & 0.2 & 0.2 \\
\hline
\end{tabular}

Notes: FI to F6: using $30 \%$ ethyl alcohol as a solvent; F2*: using $3 \%$ DMSO as a solvent. All formulas contained $20 \%$ oil phase (lecithin and Isopropyl myristate) and $80 \%$ aqueous phase (pluronic and water/solvent).

Abbreviations: PLO, pluronic-lecithin organogel; DMSO, dimethyl sulfoxide. 


\section{Characterization of the prepared PLO formulation}

\section{Fourier transform infrared spectroscopy (FTIR)}

Samples (silymarin, plain PLO gel and drug loaded PLO gel [formulation $2\{\mathrm{~F} 2\}]$ ), $1 \mathrm{mg}$ were dried, mixed with potassium bromide, compressed at a pressure of $6 \mathrm{ton} / \mathrm{cm}^{2}$ into discs, and scanned using a Fourier transform infrared spectrophotometer (PerkinElmer Inc., Waltham, MA, USA) over the range of $4,000-500 \mathrm{~cm}^{-1}$. A blank $\mathrm{KBr}$ pellet was used as a reference.

\section{Drug content}

For determining the drug content of the prepared PLO gels, a sample of $1 \mathrm{~g}$ of each formulation was transferred into a $50 \mathrm{~mL}$ volumetric flask. The volume was made up with ethyl alcohol and shaken at $100 \mathrm{rpm}$ for 10 minutes at room temperature to dissolve the drug. The samples were filtered through $0.22 \mu \mathrm{m}$ filter discs. One $\mathrm{mL}$ of the filtrate was withdrawn into a $10 \mathrm{~mL}$ volumetric flask and diluted to $10 \mathrm{~mL}$ with ethyl alcohol. The content of the drug was estimated spectrophotometrically at $\lambda \max 286 \mathrm{~nm}$ using a spectrophotometer (Spectronic Genesys ${ }^{\circledR}$, with Winspec Software; Spectronic 700, NY, USA).

\section{Viscosity and rheology study}

The viscosity and rheological studies of the samples were carried out at $25^{\circ} \mathrm{C} \pm 0.1^{\circ} \mathrm{C}$ using a Brookfield Rheometer (model DV-I+; Brookfield Engineering Laboratory, Inc, Middleboro, MA, USA). The shear stress was increased automatically by the instrument over a period of 30 seconds. The viscosities of the formulations were recorded before and after gradual increase in the angular velocity from $10 \mathrm{rpm}$ to $100 \mathrm{rpm}$. The average of three readings was used to calculate the viscosity. The relationship between shear stress and shear rate of each formulation was determined using power law described in the following equation:

$$
\tau=\mathrm{k} \gamma^{n}
$$

where $\tau$ : shear stress, $\mathrm{k}$ : consistency index, $\gamma$ : shear rate, $\mathrm{n}$ : power law index.

\section{Stability study}

Stability of the prepared formulations was also studied by monitoring the optical quality for any phase separation and viscosity over 3 months at $25^{\circ} \mathrm{C} \pm 5^{\circ} \mathrm{C}$ and around $50 \%$ relative humidity. The rheological parameters were also evaluated as previously mentioned.

\section{In vitro release studies}

In vitro release of silymarin PLO formulations was studied using phosphate buffer of $\mathrm{pH} 7.4$ containing $20 \%$ ethyl alcohol as a release medium. The release study was performed under sink conditions. A cellophane membrane of molecular weight cut-off of 6,000-8,000 Da and presoaked in the release medium was used to cover one end of a cylindrical glass tube with a surface area of $2.8 \mathrm{~cm}^{2}$. One gram of the examined formulation was placed over the inner side of the cellophane membrane and the whole assembly was immersed in $100 \mathrm{~mL}$ of the release media at $37^{\circ} \mathrm{C} \pm 0.5^{\circ} \mathrm{C}$ and subjected to shaking at 100 strokes per minute. Samples of $5 \mathrm{~mL}$ each were drawn at the specified time intervals. Each sample was replaced by an equal volume of fresh phosphate buffer. The concentration of silymarin was analyzed and validated spectrophotometrically at $\lambda \max 322 \mathrm{~nm}$.

\section{Ex vivo permeability study}

Selected formulations (F2 and *F2) were subjected to ex vivo permeability studies using excised rabbit skin. The test was carried out as mentioned in the section, In vitro diffusion study, except for using excised skin from the rabbit ear. The excised skin was cleaned by proper washing with cold running water and then treated to remove any fatty material by soaking the whole skin membranes in water at $60^{\circ} \mathrm{C}$ for 70 seconds, followed by blunt dissection and they were then folded in aluminum foil. The epidermal membranes were prepared and then mounted with the epidermal side facing the donor solution.

\section{Clinical evaluation of the prepared PLO formulations}

The clinical study was conducted according to approval from the Research Ethics Committee, Faculty of Medicine, Minia University, Minia, Egypt.

The present study was conducted on 15 patients (eight males [53.3\%] and seven females [47.7\%]) with varying degrees of AD severity, attending the outpatient clinic of Dermatology, STDs, and Andrology Department (Minia University Hospital). The age of the patients ranged from 1 year to 30 years with a mean and SD of $9.3 \pm 8.7$. The severity of AD was assessed using the SCORing Atopic Dermatitis (SCORAD) index.

The study was approved by the committee for postgraduate studies and research of Minya University. 


\section{Inclusion criteria}

Fifteen patients with $\mathrm{AD}$ were recruited by the outpatient clinic of the Department of Dermatology, STDs, and Andrology, Minia University Hospital. The inclusion criteria were:

1) All patients showing manifestation of $\mathrm{AD}$ in a bilateral distribution.

2) All ages, both sexes, and different degrees of severity of $A D$ as were assessed by the SCORAD index were included.

3) Patients received no topical or systemic treatment for at least 3 months before starting the study.

4) Written consent was obtained from all patients who participated in the present study including for the use of the photographs.

\section{Treatment of the patient groups}

The PLO containing silymarin (2.5 mg drug/dose) was applied on the right side and PLO base free from silymarin was applied on the left side. All patients were subjected to full history taking, dermatological examination, skin biopsy, and photographing as in the following sections.

\section{Patient follow-up}

Patients' symptoms and signs such as skin itching were assessed using an improvement score as follows:

1) No change $(0 \%-25 \%)$;

2) Mild improvement (26\%-50\%);

3) Moderate improvement (51\%-75\%);

4) Good result (76\%-99\%);

5) Complete cure (100\%).

\section{Photography}

Photographs of the treated lesions were obtained for all patients using a high resolution digital camera. Photographs were obtained before and 3 months after starting treatment.

\section{Skin biopsy}

Skin biopsies were obtained from patients using sterile $2 \mathrm{~mm}$ punches after informed consent.

Skin biopsies were taken from lesional skin and from apparently normal non-lesional skin.

Each biopsy was immediately fixed in 10\% formalin, embedded in paraffin, and sectioned by the ordinary microtome into $5 \mu \mathrm{m}$ thick sections. These were stained with hematoxylin and eosin and then utilized for histopathological examination. A light microscope with a built-in camera (Nikon Optiphot; Nikon Corporation, Tokyo, Japan) was used to examine and photograph these sections.

\section{Statistical analysis}

Data were collected and tested for statistically significant difference using SPSS (version 11.0; SPSS Inc., Chicago, IL, USA). The numerical data were expressed as mean \pm SD. Student's $t$-test was used to compare numerical values. The (Student's $t$-test) values were expressed in terms of $P$-values. The level of significance was determined as follows: $P>0.05=$ not significant; $P<0.05=$ significant; $P<0.001=$ highly significant.

\section{Results and discussion}

\section{Analytical method validation of silymarin}

The $\lambda$ max of silymarin in ethyl alcohol and PBS was found to be $286 \mathrm{~nm}$ and $322 \mathrm{~nm}$, respectively. The drug followed linearity in the concentration range $2-20 \mu \mathrm{g} / \mathrm{mL}$ with a correlation coefficient value of 0.998 and 0.999 in ethyl alcohol and PBS, respectively. The accuracy of the method was checked by determining percentage recovery. The percentage recovery was found to be in the range of 99.54\%-100.98\% in ethyl alcohol and 98.94\%-101.4\% in PBS, respectively. The precision of the method was studied according to intraday: interday variations, and repeatability. The percentage relative $\mathrm{SD}$ value $<2$ indicates that the method is precise. Limit of detection and limit of quantification were evaluated from signal-to-noise ratio and they were $0.3 \mu \mathrm{g} / \mathrm{mL}$ and $1 \mu \mathrm{g} / \mathrm{mL}$, respectively.

\section{Optimization of PLO formulations}

The composition of different PLO formulations of silymarin is shown in Table 1. It was found that the key factor for preparation of stable organogel was the percentage of pluronic. By increasing the ratio of pluronic from $10 \%$ to $30 \%$, the stability of the organogel was enhanced. Therefore, F1 was ruled out from our study due to instability and phase separation. Additionally, preliminary work revealed that the best ratio of the oil phase (lecithin and IPM) to the aqueous phase (pluronic solution) was 20\%:80\%. The best ratio of aqueous phase mixture was $70 \%$ water to $30 \%$ ethyl alcohol for complete solubilization of silymarin. The $\mathrm{pH}$ was found to be in the range of 6.1-6.8 reflecting suitability for topical application without any signs of irritation.

\section{Characterization of silymarin PLO}

Macroscopic examination of the prepared organogels indicated that the formulated organogels had a yellowish white to yellow color and had a good texture, free from grittiness. These results reflect acceptability by the patients. 


\section{FTIR study}

FTIR of silymarin, plain PLO gel, and drug loaded PLO gel is shown in Figure 1. FTIR of silymarin loaded PLO gel showed that the characteristic peaks of silymarin appear without any change in the spectrum of the drug. These results indicated that there was no interaction which could be detected between the drug and the components of the base.

\section{Drug content}

Drug content of the prepared formulations was found to range from $98.2 \%$ to $101.2 \%$ as shown in Table 2 . These results indicate complete distribution of the drug throughout the PLO gel base.

\section{Viscosity and rheology study}

Figure 2 shows the viscosity values of the prepared PLO formulations. The results showed that increasing the concentration of lecithin led to a significant increase in the gel viscosity (Table 2). Parsaee et al stated that long, flexible, and cylindrical giant micelles were formed upon increasing the concentration of lecithin in the formulation. ${ }^{24}$ Upon the addition of polar solvents, cylindrical reverse micelles started growing until they intertwined to form a 3-dimensional network in the organogels due to its amphiphilic nature. ${ }^{25}$

However, a significant increase in the viscosity of the PLO from 2,350 to 4,420 (cps) was observed with

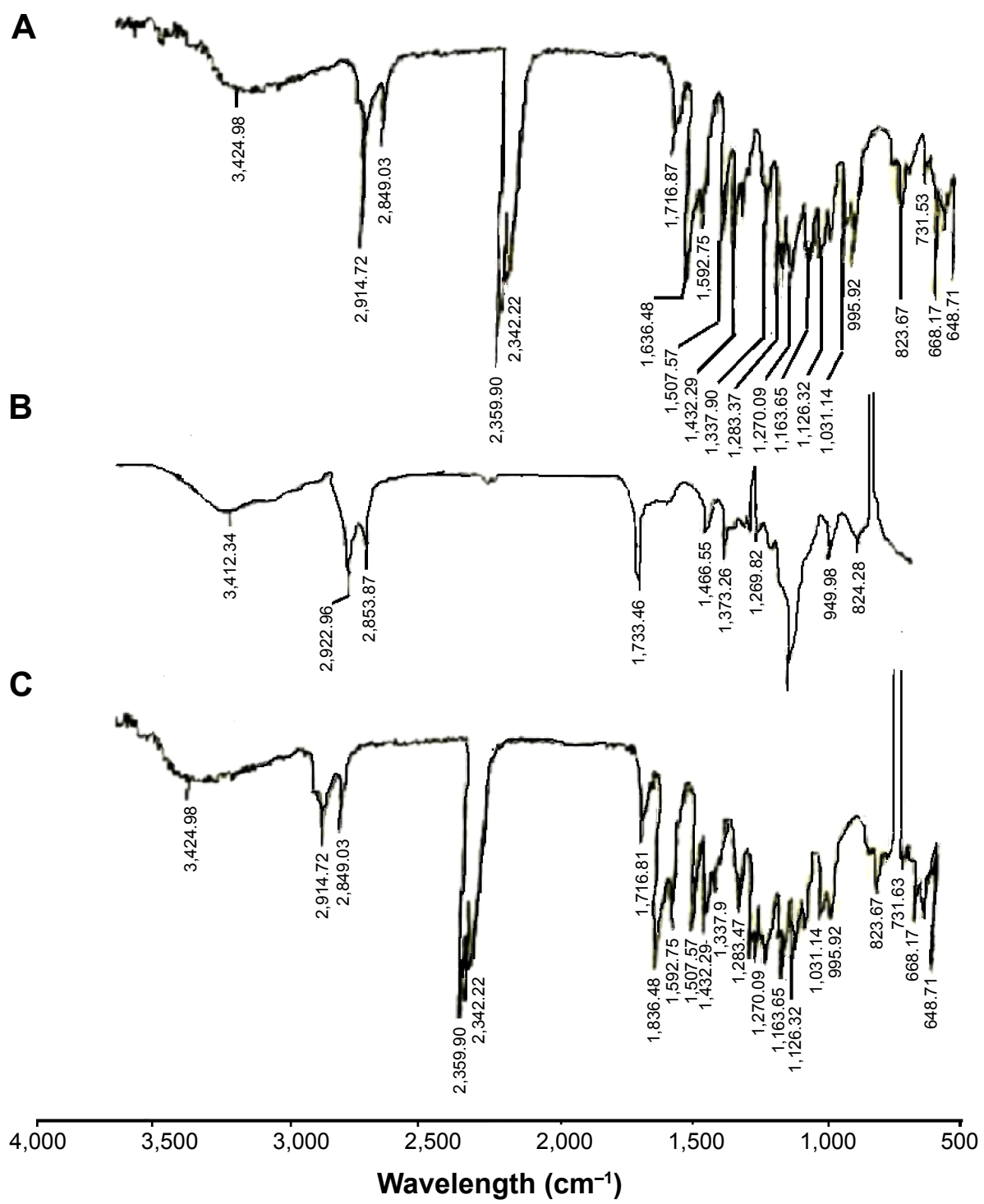

Figure I FTIR spectroscopy.

Notes: Silymarin (A), PLO gel free base (B), drug loaded PLO gel (C).

Abbreviations: FTIR, Fourier transform infrared spectroscopy; PLO, pluronic-lecithin organogel. 
Table 2 Physicochemical properties of different PLO gels loaded with silymarin

\begin{tabular}{|c|c|c|c|c|c|c|}
\hline \multirow[t]{2}{*}{ Parameters } & \multicolumn{6}{|c|}{ Formulation code } \\
\hline & $\mathbf{F 2}$ & $\mathbf{F 3}$ & $\mathbf{F 4}$ & F5 & F6 & $\mathbf{F} 2 *$ \\
\hline (\%) Drug content & 98.3 & 101.2 & 99.4 & 99.2 & 99.6 & 99.1 \\
\hline $\mathrm{pH}$ & 6.1 & 6.4 & 6.4 & 6.8 & 6.6 & 6.3 \\
\hline Drug content (\%) after 3-month storage & 98.2 & 100.9 & 99.1 & 98.5 & 99.4 & 99.2 \\
\hline Viscosity (cps) & 2,350 & 4,420 & 3,070 & 3,330 & 3,530 & 2,365 \\
\hline Viscosity after relaxation (cps) & 2,730 & 4,270 & 3,130 & 3,400 & 3,730 & 2,770 \\
\hline
\end{tabular}

Notes: F2 to F6: using 30\% ethyl alcohol as a solvent; F2*: using 3\% DMSO as a solvent.

Abbreviations: PLO, pluronic-lecithin organogel; DMSO, dimethyl sulfoxide.

increasing concentration of pluronic from $20 \%$ to $30 \%$ $(\mathrm{w} / \mathrm{v})$, respectively. These results could be ascribed to formation of a complex network. Moreover, no statistical significant difference was recorded between F2 and F2* and this indicated that type of solvent has no effect on the viscosity of the formulation.

As shown in Figure 2, all the developed formulations exhibited non-Newtonian behavior with pseudoplastic flow and shear thinning behavior. Application of the power law model to the rheological properties of the formulation enabled the calculation of the consistency $(\mathrm{k})$ and flow index (n). The values of flow index (n) were found to be less than $1(0.67)$ for the gel, confirming a shear thinning behavior of the gel. At the same time, PLO gels retained their initial viscosity at the end of the test (Table 2). This clearly indicates the better thixotropic properties which are preferable for topical use.

\section{Stability study}

Visual inspection of all prepared formulations showed no phase separation after storage for 3 months. In addition, there were no significant changes in the viscosity or rheological parameters after storage.

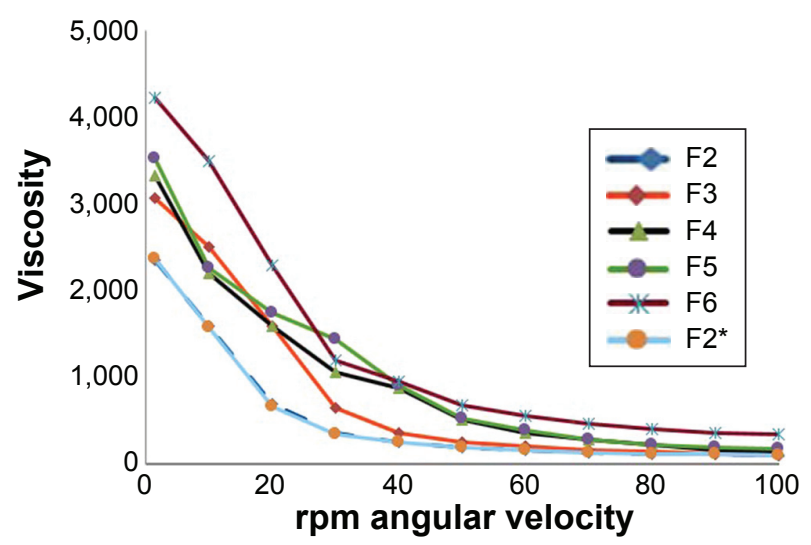

Figure 2 Rheology behaviors of different PLO gels loaded with silymarin. Notes: F2 to F6: using 30\% ethyl alcohol as a solvent; F2*: using $3 \%$ DMSO as a solvent.

Abbreviations: PLO, pluronic-lecithin organogel; DMSO, dimethyl sulfoxide.

\section{In vitro release studies and release kinetics}

In vitro release profiles of PLO formulations containing $0.2 \%(\mathrm{w} / \mathrm{w})$ silymarin are shown in Figure 3 . The results showed that the developed formulations exhibited sustained release of the drug. Data shown in Figure 3 also confirmed that the release rate of silymarin significantly decreased by increasing the lecithin ratio from $3 \%$ to $9 \%(\% \mathrm{w} / \mathrm{w})$. As mentioned before, the viscosity of the organogel was increased from 2,350 to 3,530 cps with increasing lecithin ratio from $3 \%$ to $9 \%$, respectively, which affected the release rate of the drug. As expected, at a higher lecithin concentration, there is more extensive entanglement of long cylindrical micelles, forming a network-like structure with very high viscosity, hence, a smaller amount of free drug is available for release. ${ }^{27}$

Moreover, a comparative in vitro drug release profile between F2 (20\% pluronic) and F3 (30\% pluronic) showed $12.5 \%$ and $8 \%$ after the initial 60 minutes, respectively. However, at the end of 8 hours, the drug release was found

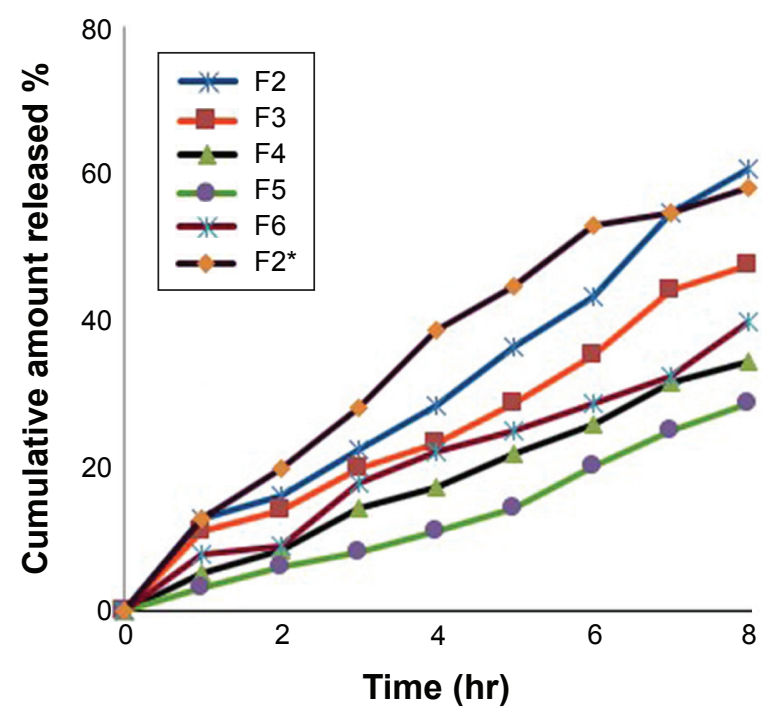

Figure 3 In vitro release of silymarin from different formulations of PLO gels. Notes: F2 to F6: using $30 \%$ ethyl alcohol as a solvent; F2*: using $3 \%$ DMSO as a solvent.

Abbreviations: PLO, pluronic-lecithin organogel; hr, hours; DMSO, dimethyl sulfoxide. 
to be $\sim 60 \%$ and $36 \%$ respectively. It was clear that increasing the ratio of pluronic led to more sustained drug release. These results could be due to two different reasons: formation of a network-like structure and high viscosity of the formulation. ${ }^{26,28}$

The conclusion which can be drawn from this study is that the most influential factor on the release rate of silymarin from the developed PLO was its viscosity. The results obtained from the in vitro test seem to emphasize the theory already described by some authors, that an inverse correlation exists between the release rate and the viscosity. ${ }^{22,26}$ In addition, there was no statistically significant difference between the percentage release of silymarin from F2 (using $30 \%$ ethyl alcohol) and F2* (using 3\% DMSO). The percentage silymarin released after 8 hours was $\sim 60 \%$ for each formulation.

Release kinetics illustrated that all the developed formulations followed zero order kinetics with correlation coefficient values ranging from 0.9758 to 0.9976 .

\section{In vitro permeability study}

Figure 4 shows the ex vivo permeability for PLOs, F2 and $\mathrm{F} 2 *$, using excised rabbit skin, compared with their in vitro release study. It was found that the permeability study was in line with the in vitro release study. These results confirm that the prepared PLO has the ability to penetrate the skin. Moreover, there was no statistically significant difference between PLOs F2 and F2*, indicating that using ethyl alcohol as a cosolvent for silymarin was successful. Ethyl alcohol was found to have the same effect as DMSO on silymarin penetration and solubilization. Therefore, PLO was successful as a novel topical formulation for silymarin.

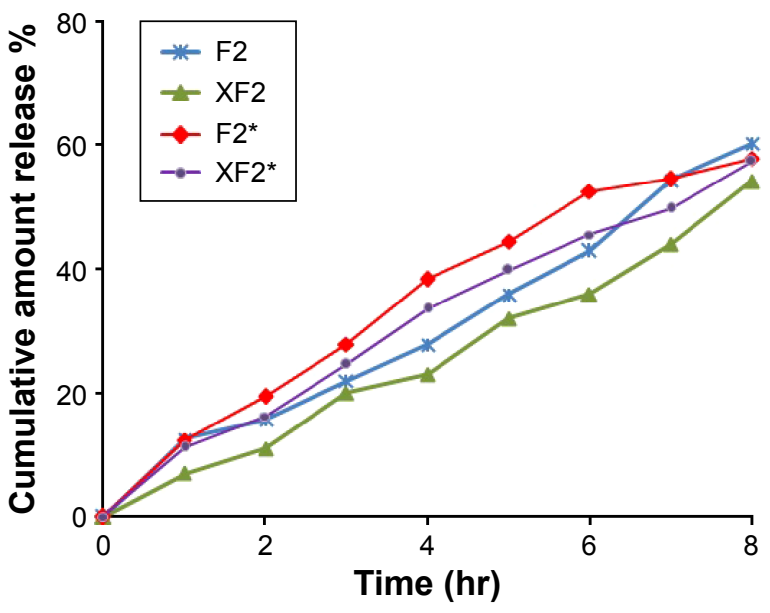

Figure 4 Ex vivo permeability release (XF2, XF2*) compared with the in vitro release $\left(F 2, F 2^{*}\right)$ of silymarin from PLO gels.

Note: F2, F2*, XF2, and XF2*: using 30\% ethyl alcohol as a solvent.

Abbreviations: PLO, pluronic-lecithin organogel; hr, hours.

\section{Clinical evaluation}

The severity of $\mathrm{AD}$ varied in different patients according to the SCORAD index. Clinical phase, severity, and distribution of lesions are tabulated in Table 3. Four cases $(26.7 \%)$ were mild, six cases (40\%) were moderate, and five cases $(33.3 \%)$ were severe.

\section{Photography}

Figure 5 shows the photographs of the lesions of some patients before and 3 months after starting treatment. It was clear that using silymarin-based PLO gel significantly improved AD in terms of redness, swelling, lichenification, and overall severity, as compared to placebo. Moreover, there was a positive improvement effect on the control side using free drug PLO gel in some patients.

\section{Patient follow-up}

Patients' signs and symptoms regarding itching were assessed using an improvement index (SCORAD) (Figure 6).

Figure 6 shows the comparison between control side and treated side regarding itching symptoms. As shown for the treated side, moderate, complete, and excellent improvement in itching was observed in 7.1\%,78.5\%, and $14.2 \%$ of patients, respectively. On the other hand, positive improvement effects were observed in 57.1\% ranging from mild (50\%) to moderate effects $(7.1 \%)$ after using drug-free PLO gel, while no change was observed in other patients $(42.8 \%)$. The difference between the two sides was statistically significant $(P=0.001)$.

\section{Histological study}

Microscopic examination of skin biopsies of AD patients before (Figure 7A) and after treatment (Figure 7B) with silymarin loaded PLO gel are shown in Figure 7. AD patients' skin histology was characterized by thickness of the epithelial layer of the skin, presence of vacuoles or holes, and increase in the inflammatory cells in the dermis layer (Figure 7A), indicating skin lichenification and inflammation of the skin due to itching. On the contrary, the treated patients' skin showed a

Table 3 Clinical phase, severity, and distribution of AD lesions among patients

\begin{tabular}{llll}
\hline Clinical phase & Infantile & Childhood & Adulthood \\
Number of cases & $5(33.3 \%)$ & $7(46.6 \%)$ & $3(20 \%)$ \\
Severity & Mild & Moderate & Severe \\
Number of cases & $4(26.7 \%)$ & $6(40 \%)$ & $5(33.3 \%)$ \\
Distribution & Face & Limbs & Trunk \\
Number of cases & $10(66.6 \%)$ & $5(33.3 \%)$ & $2(13.3 \%)$ \\
\hline
\end{tabular}

Abbreviation: $A D$, atopic dermatitis. 


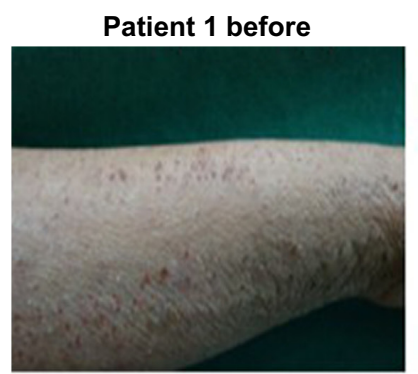

Patient 2 before

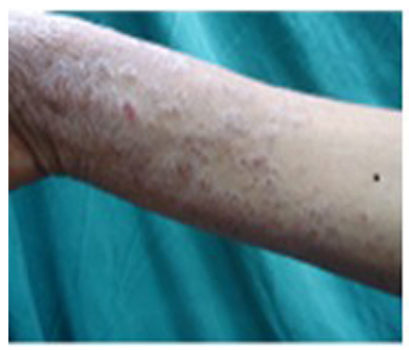

Patient 3 before

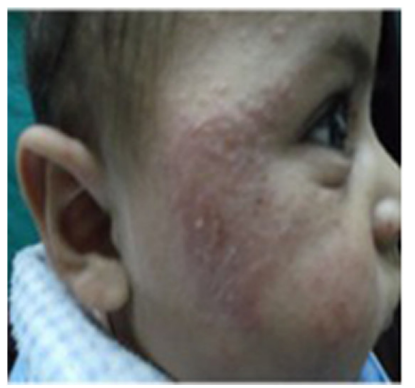

Patient 1 after

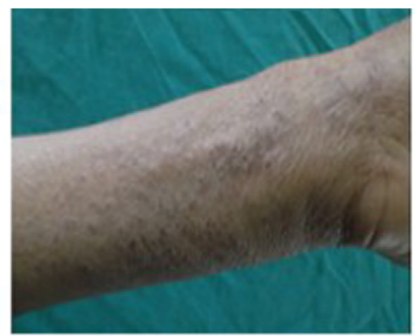

Patient 2 after

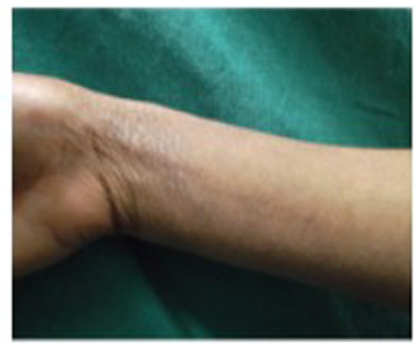

Patient 3 after

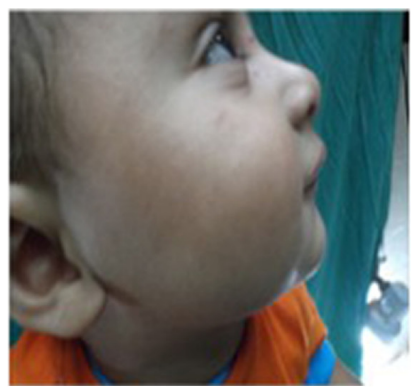

Patient 4 before

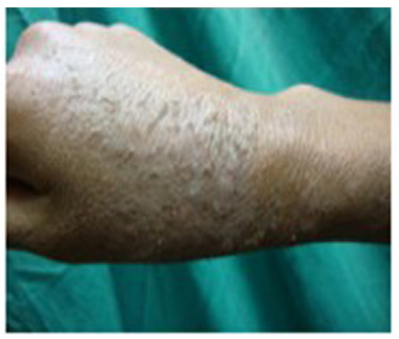

Patient 5 before

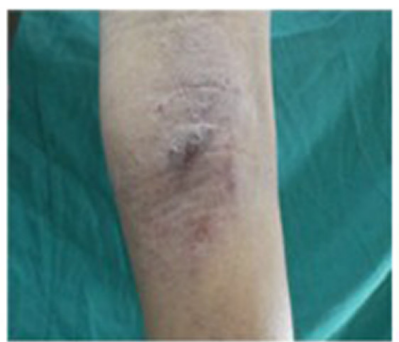

Patient 6 before

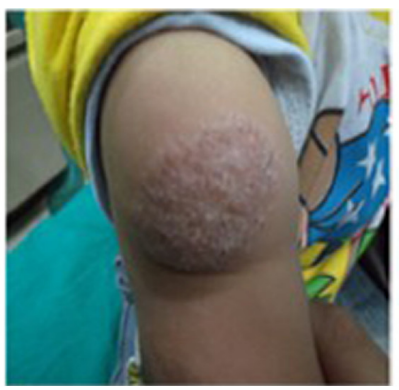

Patient 4 after

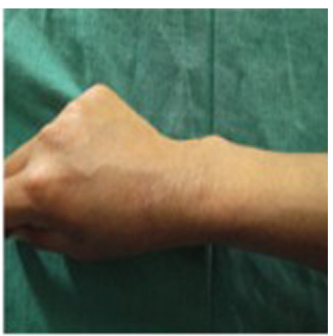

Patient 5 after

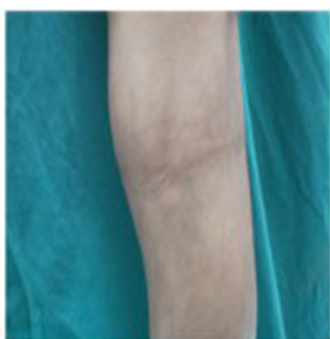

Patient 6 after

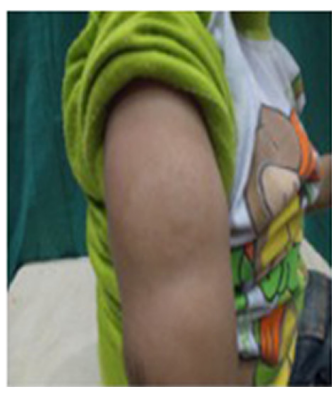

Figure 5 Photographs of different AD patients before and after treatment with silymarin loaded PLO gels. Abbreviations: PLO, pluronic-lecithin organogel; AD, atopic dermatitis.

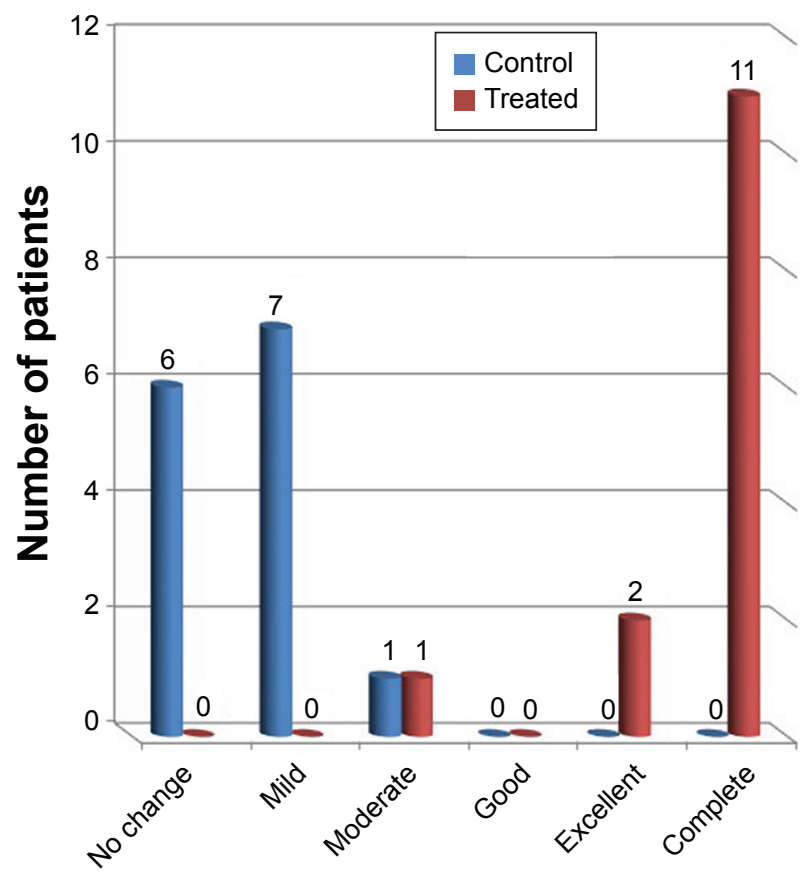

Figure 6 Comparison between control side and treated side with regards to itching. decrease in the thickness of the epidermis and a decrease in the inflammatory cells infiltrating the dermis layer (Figure 7B). These improvements can be explained according to the results obtained from a previous animal study which indicated that silymarin induced anti-oxidative, anti-inflammatory, immune modulating actions, and has been shown to support dermal regeneration. ${ }^{29-31}$ It can further be stated that the effectiveness of the topical formulation of silymarin-based PLO gel results from enhanced drug molecules' penetration despite skin lichenification. Local occlusive factor, warmth, and hydration produced by the PLO gel base are among the factors that can increase silymarin skin penetration.

Some patients in the present study who received a silymarin-free base showed mild, moderate, and complete improvement in $\mathrm{AD}$ manifestation. This could be explained by the fact that first line treatment of $\mathrm{AD}$ is hydration of skin by using an emollient, and this hydration effect was provided by the base. These results were consistent with a previous study which reported that a PLO gel base provided desired 

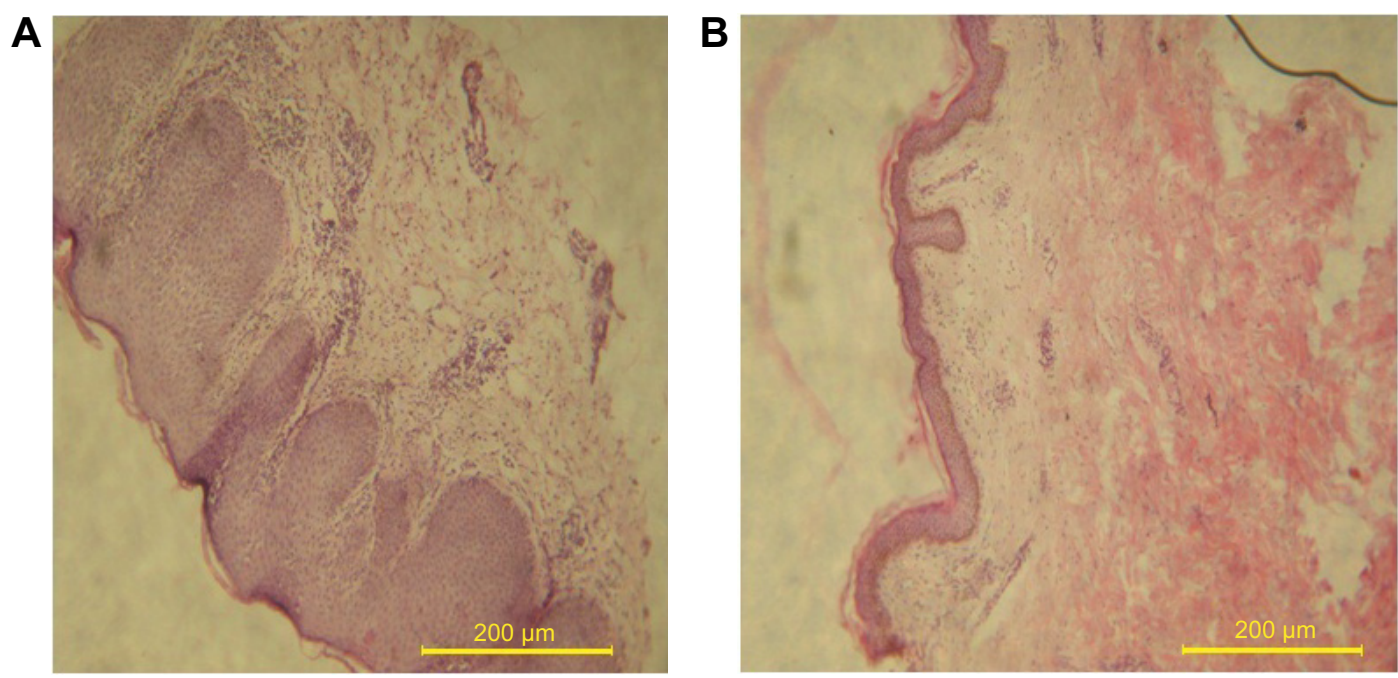

Figure 7 Patient's skin histology.

Notes: Before (A) and after (B) treatment with silymarin loaded PLO gels. Magnification= 100x; Scale bar= $200 \mu \mathrm{m}$.

Abbreviation: PLO, pluronic-lecithin organogel.

hydration to the skin, thus was effective in the improvement of eczema or psoriasis. ${ }^{32,33}$ Therefore, the PLO base could be considered as an adjuvant therapy for AD patients.

Becker-Schiebe et al used silymarin-based cream $\left(\right.$ Leviaderm $\left.^{\circledR}\right)$ for the treatment of radiodermatitis patients. ${ }^{19}$ However, cream dosage form is the most suitable vehicle for the moist and subacute weeping lesions. Moreover, emulsifiers must be added to stabilize cream, thus having the potential to sensitize and cause allergic reactions. On the other hand, dry, fissured, and lichenified skin diseases need an occlusive base due to its moisturizing effect.

\section{Conclusion}

Silymarin loaded PLO gel was successfully prepared. The high penetrating ability and hydration effect of the base provided a significant improvement in the signs and symptoms of $\mathrm{AD}$ patients.

Therefore, it could be concluded that silymarin loaded PLO gel may be introduced as a novel topical formulation of silymarin.

\section{Disclosure}

The authors have no conflicts of interest to disclose in this work.

\section{References}

1. Darsow U, Raap U, Ständer SR. In: Carstens E, Akiyama T, editors. Itch: Mechanisms and Treatment. Boca Raton: CRC Press; 2014:832-836.

2. Ring J, Przybilla B, Ruzicka T. Handbook of atopic eczema. 2nd ed. New York: Springer-Verlag Berlin Heidelberg; 2006.
3. Friedmman PS, Holden CA. Atopic dermatitis. In: Burns T, Breathnach S, Cox N, Griffiths C, editors. Rook's Textbook of Dermatology. 7th ed. Oxford, England: Blackwell Sciences; 2008:755-786.

4. Charman C. Clinical evidence: atopic eczema. BMJ. 1999;318(7198): $1600-1604$.

5. Sudilovsky A, Muir JG, Bocobo FC. A comparison of single and multiple applications of halcinonide cream. Int $J$ Dermatol. 1981;20(9):609-613.

6. Darsow U, Lübbe J, Taïeb A, et al. Position paper on diagnosis and treatment of atopic dermatitis. J Eur Acad Dermatol Venereol. 2005; 19(3):286-295.

7. Haeck IM, Rouwen TJ, Mik LT, Bruin-Weller MS, BruijnzeelKoomen CA.Topical corticosteroids in atopic dermatitis and the risk of glaucoma and cataracts. J Am Acad Dermatol. 2011;64(2):275-281.

8. Hajar T, Leshem YA, Hanifin JM, et al. A systematic review of topical corticosteroid withdrawal ("steroid addiction") in patients with atopic dermatitis and other dermatoses. JAm Acad Dermatol. 2015;72(3):541e-549e. e2.

9. O’Hara M, Kiefer D, Farrell K, Kemper K. A review of 12 commonly used medicinal herbs. Arch Fam Med. 1998;7(6):523-536.

10. Zhao J, Lahiri-Chatterjee M, Sharma Y, Agarwal R. Inhibitory effect of a flavonoid antioxidant silymarin on benzoyl peroxide-induced tumor promotion, oxidative stress and inflammatory responses in SENCAR mouse skin. Carcinogenesis. 2000;21(4):811-816.

11. Gazak R, Walterova D, Kren V. Silybin and silymarin - new and emerging applications in medicine. Curr Med Chem. 2007;14(3):315-338.

12. Singh RP, Agarwal R. Cosmeceuticals and Silibinin. Clin Dermatol. 2009;27(5):479- 484

13. Dehmlow C, Erhard J, de Groot H. Inhibition of Kupffer cell functions as an explanation for the hepatoprotective properties of silibinin. Hepatology. 1996;23(4):749-754.

14. Rasul A, Naveed A, Ali A, et al. Assessment of anti erythmic and skin whitening effects of milk thistle extract. African Journal of Pharmacy and Pharmacology. 2011;520(20):2306-2309.

15. Svobodová A, Walterová D, Psotová J. Influence of silymarin and its flavonolignans on $\mathrm{H}(2) \mathrm{O}(2)$-induced oxidative stress in human keratinocytes and mouse fibroblasts. Burns. 2006;32(8):973-979.

16. Toklu HZ, Tunal1-Akbay T, Erkanl G, Yuksel M, Ercan F, Sener G. Silymarin, the antioxidant component of Silybum marianum, protects against burn-induced oxidative skin injury. Burns. 2007;33(7):908-916. 
17. Ashkani-Esfahani S, Emami Y, Esmaeilzadeh E, et al. Silymarin enhanced fibroblast proliferation and tissue regeneration in full thickness skin wounds in rat models; a stereological study. Journal of the Saudi Society of Dermatology \& Dermatologic Surgery. 2013;17(1):7-12.

18. Han $\mathrm{MH}$, Yoon WK, Lee H, et al. Topical application of silymarin reduces chemical-induced irritant contact dermatitis in BALB/c mice. Int Immunopharmacol. 2007;7(13):1651-1678.

19. Becker-Schiebe M, Mengs U, Schaefer M, Bulitta M, Hoffmann W. Topical use of a silymarin-based preparation to prevent radiodermatitis: results of a prospective study in breast cancer patients. Strahlenther Onkol. 2011;187(8):485-491.

20. Gillet A, Lecomte F, Hubert P, Ducat E, Evrard B, Piel G. Skin penetration behaviour of liposomes as a function of their composition. Eur J Pharm and Biopharm. 2011;79(1):43-53.

21. Josef E, Zilberman M, Bianco-Peled H. Composite alginate hydrogels: An innovative approach for the controlled release of hydrophobic drugs. Acta Biomater. 2010;6(12):4642-4649.

22. Spada G, Gavini E, Cossu M, Rassu G, Carta A, Giunchedi P. Evaluation of the effect of hydroxypropyl- $\beta$-cyclodextrin on topical administration of milk thistle extract. Carbohydr Polym. 2013;92(1):40-47.

23. Kumar R, Katare OP. Lecithin organogels as a potential phospholipid structured system for topical drug delivery: a review. AAPS PharmSciTech. 2005;6(2):E298-E310.

24. Parsaee S, Sarbolouki MN, Parniapour M. In-vitro release of diclofenac dethylammnium from lipid-based formulations. Int J Pharm. 2002; 241(1):185-190.

25. Peinzes T, Csoika I, Eros I. Rheological analysis of the structural properties effecting the percutaneous absorption and stability in pharmaceutical organogels. Rheol Acta. 2004;43:457-463.
26. Balata G, El Nahas HM, Radwan S. Propolis organogel as a novel topical delivery system for treating wounds. Drug Deliv. 2014;21(1): $55-61$.

27. Surjyanarayan M, Snigdha SM, Krutika KS. Lecithin stabilized organogel: design and development for topical application of clobetasol propionate. Int J Pharm Tech Res. 2010;2(2):1133-1138.

28. Stationwala R, Patidar A, Main P. Transdermal delivery of Iornoxicam from pluronic lecithin organogel. Int J Chem Pharm Sci. 2011; 2(2):32-37.

29. Piscitelli SC, Formentini E, Burstein AH, Alfaro R, Jagannatha S, Falloon J. Effect of milk thistle on the pharmacokinetics of indinavir in healthy volunteers. Pharmacotherapy. 2002;22(5):551-556.

30. Johnson VJ, He Q, Osuchowski MF, Sharma RP. Physiological responses of a natural antioxidant flavonoid mixture, silymarin, in BALB/c mice: III. Silymarin inhibits T-lymphocyte function at low doses but stimulates inflammatory processes at high doses. Planta Med. 2003;69(1):44-49.

31. Kang JS, Yoon WK, Han MH, et al. Inhibition of atopic dermatitis by topical application of silymarin in NC/Nga mice. Int Immunopharmacol. 2008;8(10):1475-1480.

32. Almeida H, Amaral MH, Lobao P, Lobo JM. Pluronic ${ }^{\circledR}$ F-127 and Pluronic Lecithin Organogel (PLO): main features and their applications in topical and transdermal administration of drugs. J Pharm Pharm Sci. 2012;15(4):592-605.

33. Bhatia A, Singh B, Raza K, Wadhwa S, Katare O. Tamoxifen-loaded lecithin organogel (LO) for topical application: Development, optimization and characterization. Int J Pharm. 2013;444(1-2):47-59.
Drug Design, Development and Therapy

\section{Publish your work in this journal}

Drug Design, Development and Therapy is an international, peerreviewed open-access journal that spans the spectrum of drug design and development through to clinical applications. Clinical outcomes, patient safety, and programs for the development and effective, safe, and sustained use of medicines are a feature of the journal, which

\section{Dovepress}

has also been accepted for indexing on PubMed Central. The manuscript management system is completely online and includes a very quick and fair peer-review system, which is all easy to use. Visit http://www.dovepress.com/testimonials.php to read real quotes from published authors. 